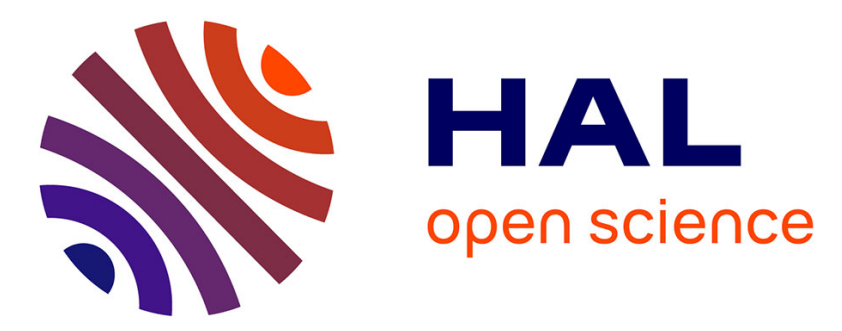

\title{
Dessin de la main et habiletés numériques chez des enfants d'âge préscolaire.
}

Nathalie Bonneton-Botté, Hélène Hili, Fanny de La Haye, Yvonnick Noël

\section{To cite this version:}

Nathalie Bonneton-Botté, Hélène Hili, Fanny de La Haye, Yvonnick Noël. Dessin de la main et habiletés numériques chez des enfants d'âge préscolaire.. Canadian Journal of Behavioural Science, 2015, 47 (3), pp.207-215. 10.1037/a0039037 . hal-01625443

\section{HAL Id: hal-01625443 \\ https://hal.science/hal-01625443}

Submitted on 27 Oct 2017

HAL is a multi-disciplinary open access archive for the deposit and dissemination of scientific research documents, whether they are published or not. The documents may come from teaching and research institutions in France or abroad, or from public or private research centers.
L'archive ouverte pluridisciplinaire HAL, est destinée au dépôt et à la diffusion de documents scientifiques de niveau recherche, publiés ou non, émanant des établissements d'enseignement et de recherche français ou étrangers, des laboratoires publics ou privés. 


\title{
Dessin de la main et habiletés numériques chez des enfants d'âge préscolaire
}

\author{
Nathalie Bonneton-Botté, Hélène Hili et \\ Fanny De La Haye \\ École supérieure du professorat et de l'éducation de Bretagne
}

\author{
Yvonnick Noël \\ Université de Rennes 2
}

\begin{abstract}
Cette étude propose d'étudier les relations entre les représentations manuelles et digitales et les habiletés numériques au travers du dessin de la main. Soixante-deux enfants âgés de 4 ans à 5 ans 6 mois ont reçu pour consigne de dessiner une main et de réaliser deux tâches numériques. La première consiste pour l'enfant à produire une collection de jetons identique à celle qui lui est proposée (tâche de correspondance terme à terme). La seconde tâche teste la maitrise du principe de non pertinence de l'ordre. L'analyse montre que les stratégies graphiques les plus élaborées impliquent plus fréquemment une réussite à la tâche de correspondance terme à terme.
\end{abstract}

Mots-clés : représentation sensori-motrice manuelle, dessin, habiletés numériques, développement.

La relation existant entre les mains et les habiletés numériques a été abordée au travers de différents champs disciplinaires. En archéologie, l'utilisation des doigts pour assister le calcul a été identifiée comme une méthode très ancienne (Williams \& Williams, 1995). En neuropsychologie, une proximité topographique entre les zones accueillant les représentations des doigts et des mains et celles contribuant au traitement des nombres (Gertsmann, 1940; Dehaene, Piazza, Pinel, Cohen, 2003) est aujourd'hui attestée. Cette proximité expliquerait pourquoi tant des adultes que des enfants (Kinsbourne \& Warrington, 1963; Rourke, 1993) peuvent présenter simultanément des troubles de la perception digitale et des troubles affectant le traitement du nombre. Une hypothèse fréquemment retenue en neuropsychologie est celle d'une simple proximité spatiale entre les deux zones concernées, sans relation fonctionnelle particulière entre les habiletés manuelles et numériques (Marinthe, Fayol \& Barrouillet, 2001). Néanmoins, l'existence d'une éventuelle relation fonctionnelle entre ces deux habiletés est aussi régulièrement envisagée. Récemment, les résultats obtenus par Andres, Michaux et Pesenti (2012) alimentent cette thèse fonctionnelle en montrant une activation des mêmes régions cérébrales lorsque le sujet adulte réalise des tâches de discrimination des doigts et des tâches d'addition, tandis que cette activation est moins significative lorsque la tâche mathématique proposée concerne les multiplications, opération connue pour être
Nathalie Bonneton-Botté et Fanny De La Haye, École supérieure du professorat et de l'éducation de Bretagne et Centre de recherches en psychologie, cognition et communication, France; Hélène Hili, École supérieure du professorat et de l'éducation de Bretagne, France; Yvonnick Noël, Centre de recherches en psychologie, cognition et communication, Université Rennes 2, France.

Toute correspondance concernant le présent article doit être adressée à : Nathalie Bonneton-Botté, École supérieure du professorat et de l'éducation de Bretagne, Laboratoire CRPCC, EA 1285, 1, rue Théodule Ribot, 22000 Saint-Brieuc, France. Courriel : nathalie.bonneton@espe-bretagne.fr généralement résolue par une récupération du résultat en mémoire à long terme. La relation entre les habiletés manuelles et la capacité à manipuler mentalement des quantités est aussi étayée par quelques travaux en psychologie développementale. Les conclusions alimentent fréquemment l'hypothèse d'une relation fonctionnelle entre gnosies digitales et performances arithmétiques (Butterworth, 1999). Ces recherches montrent généralement que les enfants qui recourent le plus souvent aux doigts pour calculer dans les classes préscolaires sont ceux qui présenteront les meilleures performances en calcul en deuxième année d'école primaire (Jordan, Kaplan, Ramineni \& Locuniak, 2008). L'utilisation des doigts permettrait au jeune enfant de se représenter les quantités sur un mode analogique, avant que les représentations symboliques ne soient disponibles, et d'opérer des calculs sur ces représentations (Siegler \& Shrager, 1984). Les doigts sont aussi fréquemment utilisés par les jeunes enfants pour accompagner l'oralisation de la chaîne numérique (Sato \& Lalain, 2008). Di Luca et Pesenti (2011) soulignent que l'utilisation d'une séquence de mouvements ordonnée et stable lors du comptage permet à l'enfant de mémoriser les éléments déjà comptés en s'appuyant sur la correspondance terme à terme entre les doigts levés et les objets comptés. Ils supposent aussi que cette stratégie facilite la compréhension et le développement du concept de nombre, notamment ses propriétés cardinale et ordinale. Le recours aux doigts ne semble cependant pas concerner tous les enfants de la même façon. Un suivi longitudinal d'élèves réalisé par Jordan et Levine (2009) a aussi montré que les enfants de catégorie socioprofessionnelle défavorisée utilisent moins fréquemment leurs doigts en dernière année de maternelle pour résoudre de petites opérations et montrent de moins bonnes performances deux années plus tard sur ce même type d'épreuve. Les auteurs de ces travaux suggèrent d'enseigner explicitement le recours aux doigts aux enfants de niveau préscolaire. L'hypothèse d'une relation fonctionnelle entre la représentation mentale de la main et les habiletés arithmétiques s'est aussi renforcée grâce aux études longitudinales associant une évaluation des gnosies digitales à des épreuves numériques. Une étude datant 
BONNETON-BOTTÉ, HILI, DE LA HAYE ET NOËL

de 1998 montre que les capacités perceptivo-tactiles de 300 enfants de 5 et 6 ans présentant un développement ordinaire prédisent mieux la réussite en calcul que leur niveau de développement intellectuel (Marinthe, Fayol \& Barrouillet, 1999). Trois ans plus tard, la même équipe de chercheurs montrera que cette relation se maintient dans le temps pour les mêmes enfants. Ils préciseront aussi la nature de la relation en montrant que les gnosies digitales, au moins pour les enfants âgés de 8 ans, prédisent les performances d'épreuves numériques non verbales nécessitant de se représenter et de manipuler des quantités et non celles d'épreuves faisant appel à une mémoire verbale des faits arithmétiques. Cette relation a été retrouvée par Noël (2005), qui montrera qu'entre 6 et 7 ans, les scores de gnosies digitales prédisent mieux les habiletés numériques que les habiletés de lecture. Les conclusions de ces recherches apporteraient un argument supplémentaire en faveur de l'existence d'un patron de dyscalculie développementale de type perceptivo-motrice (Marinthe et al., 2001; Rourke, 1993). L'état actuel de nos connaissances ne nous permet pas de trancher entre les hypothèses localisationniste et fonctionnelle (Noël, 2005; Crollen, Seron \& Noël, 2011). Comme le suggère Fayol (2012), des études montrant l'effet bénéfique d'entraînement aux gnosies digitales seraient nécessaires pour alimenter ces thèses. GraciaBafalluy et Noël (2008) ont proposé ce type d'entraînement à 33 élèves de première année primaire ayant de faibles performances en gnosie digitale ainsi qu'à 14 élèves présentant de bonnes performances. Dans cette étude, les auteurs questionnent la nature du lien qui pourrait exister entre la qualité de la représentation manuelle et les habiletés numériques. Ils supposent que s'il existe un lien fonctionnel entre ces deux habiletés, alors un entraînement visant une meilleure reconnaissance et une meilleure discrimination digitale devrait avoir un impact positif sur les habiletés numériques. À l'issue des huit semaines d'entraînement, le groupe expérimental obtient les meilleures performances aux tests de gnosies et à certaines épreuves mathématiques (représentation de quantités à l'aide de doigts et tâches de subitizing) pour lesquels il ne s'est pourtant pas exercé. Par ailleurs, les auteurs constatent aussi un effet bénéfique de cet entraînement sur la représentation interne des doigts et de la main évaluée par un dessin de la main réalisé par l'enfant, tandis que le dessin du bonhomme réalisé avant et après entraînement n'évolue pas significativement. Il nous semble que ce dernier résultat mériterait des investigations supplémentaires. En effet, s'il doit exister une relation entre la qualité $\mathrm{du}$ dessin de la main et les performances en gnosie digitale, on comprend mal pourquoi les groupes constitués au départ de cette étude sur la base de performances gnosiques hautes ou basses, ne présentent pas de différence quant à la qualité du dessin de la main fournie au prétest. Il nous semble au contraire que la relation fonctionnelle hypothétique supposée entre gnosie digitale et habileté numérique devrait être observée au travers du dessin de la main. Par cette recherche, nous aimerions apporter deux types de contributions.

\section{Objectifs de l'étude}

Premièrement, nous souhaitons observer et décrire les capacités des enfants à dessiner une main sans modèle au cours de la cinquième année. Pour le dessin du bonhomme, les différentes étapes sont bien connues (Goodenough, 1926; Luquet, 1927). En revanche, il n'existe pas, à notre connaissance, d'études analysant de façon systématique les caractéristiques du dessin de la main chez des enfants d'âge préscolaire. Contrairement au dessin du bonhomme, qui peut probablement être réalisé à partir d'une procédure algorithmique plutôt qu'à partir d'une représentation interne du corps (Picard \& Baldy, 2011), le dessin de la main n'est pas une tâche communément demandée au jeune enfant. De ce fait, sa représentation graphique devrait mieux refléter la représentation interne de la main. Dans le cadre de cette étude, nous observerons, à l'instar de Gracia-Bafalluy et Noël (2008), la qualité globale de la production graphique, c'est-à-dire ici la capacité de l'enfant à représenter fidèlement les différentes parties constitutives de la main (la paume et les cinq doigts). Cependant, étudier la capacité de l'enfant à produire un dessin réaliste ne nous semble pas suffisant. Pour Vinter et Marot (2003), les stratégies graphiques révèlent la nature des représentations mentales et, plus particulièrement, la taille des unités cognitives. De ce fait, nous nous proposons d'étudier la planification de l'acte graphique au travers de la capacité de l'enfant à produire un dessin par un tracé continu ou au contraire en le réalisant segment par segment. Vinter, Picard et Fernandes (2008) ont pu montrer que cette capacité à organiser la séquence de mouvements de l'acte graphique en se référant à un modèle interne global apparaissait à partir de 6 ans lorsqu'il s'agit de copier des formes géométriques. À 4 et 5 ans, l'enfant à qui on demande la copie de formes simples serait enclin à planifier localement son tracé (segment par segment).

Deuxièmement, nous chercherons à décrire les relations qui caractérisent les performances au dessin de la main et aux épreuves numériques chez un même enfant. Notre étude s'intéressera aux compétences d'enfants sur la période qui s'étend de 4 ans à 5 ans 6 mois. Au cours de cette période, l'enfant est théoriquement en mesure de recourir aux représentations graphiques pour représenter le réel et, comme nous l'avons mentionné précédemment, la capacité de tracer en continu à partir d'un modèle interne émerge. Du point de vue des habiletés numériques, c'est aussi une période essentielle de mise en relation des représentations analogiques et symboliques des quantités (Fayol, 2012). Notre attention se centrera sur la relation entre certaines caractéristiques du dessin et la représentation du nombre chez l'enfant dans sa composante analogique (la correspondance terme à terme) et conceptuelle (la non pertinence de l'ordre).

En nous appuyant sur les travaux de Vinter et Marot (2003) et de Vinter et al. (2008), notre première hypothèse suppose que l'étude conjointe des caractéristiques figuratives et syntaxiques (nous entendons ici l'organisation du tracé, sa segmentation ou sa continuité) du dessin de la main révèlera une diversité de stratégies au cours de la période étudiée. Nous affirmons avec les auteurs précédemment cités que ces stratégies reflètent la nature des représentations mentales de la main. Aussi cette analyse devraitelle permettre de distinguer les enfants capables d'organiser le tracé à partir d'une représentation interne globale de la main (la main est conçue comme un tout) de ceux qui organisent le tracé à partir de représentations locales (la paume puis les doigts). Cette analyse intégrera aussi la capacité de l'enfant à prévoir et à représenter cinq doigts sur son dessin. Aussi notre première hypothèse, exploratoire, nous conduira-t-elle à caractériser la nature et la fréquence des stratégies graphiques des enfants de cette période lorsqu'il s'agit pour eux de dessiner une main sans modèle.

Notre seconde hypothèse avance que l'on observera un lien entre la production graphique caractérisée par trois indicateurs 
(nombre de doigts, score global, planification) et les habiletés numériques des jeunes enfants. Si comme le supposent GraciaBafalluy et Noël (2008), le dessin de la main de l'enfant traduit ses représentations mentales et si ces représentations sont au service d'habiletés numériques, alors les enfants qui créent les productions graphiques les plus abouties devraient aussi être les enfants les plus performants aux tâches numériques. Cette relation sera éprouvée à partir de deux tâches numériques accessibles aux enfants de cette période. Premièrement, nous étudierons cette relation au moyen de la correspondance terme à terme, qui renvoie à la capacité de produire deux collections équivalentes par la mise en correspondance de chaque élément d'une collection avec un élément et un seul de l'autre collection. Cette capacité de correspondance terme à terme est essentielle à la compréhension de la cardinalité définie comme la capacité d'identification précise de la taille d'une collection (Fayol, 2012). Deuxièmement, cette relation supposée sera analysée à partir d'une épreuve plus verbale et conceptuelle, la non-pertinence de l'ordre. La réussite à une tâche de non-pertinence de l'ordre fournit des informations sur la conceptualisation par l'enfant des relations entre comptage et nombre, c'est-à-dire sa capacité à coordonner les propriétés cardinale et ordinale du nombre. En proposant cette deuxième tâche, plus conceptuelle, nous aimerions questionner la nature du lien éventuel qui relie la main et le nombre. Le lien supposé dans la littérature ne concerne-t-il le nombre que dans sa composante analogique ou se retrouve-t-il dans une composante plus conceptuelle ?

\section{Méthode}

\section{Population}

L'échantillon composé de 62 enfants âgés, en moyenne, de 4 ans 9 mois (entre 4 ans et 5 ans 6 mois) est constitué de 31 garçons et 31 filles. L'échantillon a été composé à partir des populations de quatre écoles de type périurbain non catégorisées en zone d'éducation prioritaire, il n'existe donc pas de surreprésentation des milieux socio-économiques défavorisés. Au regard des dossiers scolaires, les enfants inclus dans cette étude ne présentent pas de trouble avéré ni de retard de développement.

\section{Recueil des données}

Les enfants ont été rencontrés dans leur école afin de produire le dessin d'une main ainsi que deux épreuves appartenant au domaine des habiletés numériques. Les épreuves se déroulent le même jour, en début de matinée, et sont proposées par le même expérimentateur. Les passations se font à l'école de l'enfant, et les épreuves sont dispensées individuellement dans une pièce calme.

\section{Epreuves et critères retenus}

Dessin de la main : consigne de l'épreuve. L'expérimentateur propose à l'enfant de dessiner une main : «Peux-tu dessiner une main ? » Souhaitant que la situation soit la plus écologique possible, aucune consigne supplémentaire n'est donnée. Conformément à la situation décrite par Gracia-Bafalluy et Noël (2008), le crayon est posé sur la feuille de telle sorte que l'enfant puisse engager la tâche avec sa main dominante. Lorsque certains enfants ont voulu utiliser l'autre main pour en faire un contour, l'expérimentateur leur a alors expliqué que cela n'était pas possible et qu'il devait le faire sans modèle. Étonnamment, nous n'avons pas observé d'enfant utilisant l'autre main comme modèle. AQ: 2

\section{Dessin de la main : variables dépendantes retenues et identification de stratégies graphiques}

Score reflétant la qualité du dessin de la main. Ce score reprend certains des indicateurs étudiés par Gracia-Bafalluy et Noël (2008) : taille relative des doigts, opposition pouce-doigts, taille relative paume-doigts, doigts attachés à la paume, différenciation paume-doigts. Chaque critère correspond à un point. Le score maximum est de 5 .

Nombre de doigts. Le nombre de doigts est codé 1 ou 0 selon que l'enfant représente ou non le nombre exact de doigts.

Planification du dessin. Vinter (1999) et Vinter et Marot (2003) décrivent à partir de tâches de copie de formes simples une période, entre 4 et 5 ans, au cours de laquelle les dessins sont planifiés segment par segment (organisation locale). À partir de 6 ans, la planification globale du dessin de la figure apparaîtrait (organisation globale). Dans notre étude, il nous a semblé intéressant de caractériser le degré de planification de la routine graphique des enfants : se représentent-ils la main comme étant composée de plusieurs segments juxtaposés - la paume est dessinée puis des doigts sont ajoutés ? Ou la voient-ils comme un ensemble - la réalisation des doigts étant planifiée dès le début de la routine ? Lorsque le tracé de la main est réalisé en continu, le score est de 1, si les doigts (un ou plusieurs) sont ajoutés progressivement au dessin de la paume, le score est de 0 .

La nature et la fréquence d'occurrence des stratégies graphiques seront étudiées en considérant simultanément le nombre de doigts présents sur le dessin et la capacité de planification locale ou globale. Nous avons donc catégorisé les productions en 4 types de stratégies : la première, la plus élémentaire, caractérise des productions planifiées localement et avec une méconnaissance du nombre de doigts à représenter $(\mathbf{D}-\mathbf{P}-)$. La seconde concerne des productions non planifiées, mais qui aboutissent à une main réaliste à cinq doigts $(\mathbf{D}+\mathbf{P}-)$. La troisième concerne les productions qui ont été réalisées à partir d'un modèle interne global de la main, mais qui échouent à faire figurer les cinq doigts $(\mathbf{P}+\mathbf{D}-)$. La quatrième représente les productions les plus élaborées puisque le tracé est réalisé à partir d'un modèle interne global de la main et que la production est fidèle du point de vue du nombre de doigts attendus $(\mathbf{P}+\mathbf{D}+)$.

\section{Habiletés numériques : description des épreuves et variables dépendantes retenues}

Épreuve de correspondance terme à terme. Nous souhaitions recourir à une épreuve pouvant rendre compte de la capacité de l'enfant à se représenter une quantité de façon analogique. Il fallait que cette épreuve soit accessible dès la cinquième année et qu'elle ne soit pas verbale. Nous avons considéré que l'épreuve de correspondance terme à terme pouvait être pertinente à cet âge (Van Nieuwenhoven, 1999). Il s'agit pour l'enfant de reproduire une collection à partir d'une collection témoin de six jetons : « Peux-tu mettre ici le même nombre de jetons, pour qu'il n'y en ait pas plus pas moins, pour qu'il y en ait pareil. » Le score attribué est de 1 si l'enfant réussit à produire une collection quantitativement équivalente. 
BONNETON-BOTTÉ, HILI, DE LA HAYE ET NOËL

Épreuve de non-pertinence de l'ordre. À cette première épreuve, nous avons associé une seconde épreuve fondée sur une connaissance conceptuelle de la non-pertinence de l'ordre en nous inspirant des épreuves proposées par Gelman et Meck (1983) et de Geary et Hoard (2002). Le principe de non-pertinence de l'ordre est la compréhension par l'enfant que l'ordre dans lequel les éléments d'une collection sont énumérés n'affecte pas le résultat du comptage. Dans la situation ici proposée, un personnage en plastique compte six jetons devant l'enfant (par l'entremise de l'expérimentateur) en partant de la droite, puis recommence en partant de la gauche. Un troisième essai consiste à faire compter le personnage à partir du troisième jeton. Il s'agit cette fois de vérifier que l'enfant n'a pas élaboré une pseudorègle consistant à croire que les éléments doivent être comptés en respectant la contiguiité. L'expérimentateur demande à l'enfant si le personnage a bien compté et de justifier sa réponse. Toutes les justifications faisant référence au cardinal (« oui, c'est bon, ça fait toujours six ») ou explicitant la non-pertinence de l'ordre (« ce n'est pas grave de compter dans ce sens-là ») ont été considérées comme justes. Au contraire, chaque fois que l'enfant a justifié en énonçant une pseudo-règle de comptage ("non, c'est faux, il n'a pas le droit de compter dans ce sens"), nous avons considéré que le principe n'était pas acquis. Le score attribué est de 0 ou 1 , selon que l'on considère que la non-pertinence de l'ordre est acquise ou non. Pour obtenir le score de 1 point, il faut donc réussir aux trois essais.

\section{Analyses statistiques des données}

Une première analyse descriptive présentera la fréquence des stratégies observées à la tâche de dessin et la fréquence de réussite aux épreuves numériques. Puisque nos données sont principalement binaires et que notre hypothèse prévoit l'existence d'une relation orientée entre les performances au dessin de la main et aux tâches numériques, nous utiliserons l'analyse statistique implicative (ASI) comme outil d'analyse. Contrairement aux études corrélationnelles ou au khi carré habituellement utilisés pour tester la relation entre variables, l'ASI permet d'analyser le sens de la relation entre deux variables $a$ et $b$ de telle sorte que «si la variable $a$ est observée dans la population, alors on note une propension à observer la variable $b$ » (Gras \& Kuntz, 2008). Enfin, le score reflétant la qualité du dessin n'étant pas une variable binaire, il sera étudié à partir d'une ANOVA.

Malgré l'absence d'hypothèses relatives à l'effet du sexe dans cette étude, nous vérifierons que cette variable n'a pas d'incidence sur les variables étudiées.

\section{Résultats}

\section{Caractérisation du dessin de la main}

Le Tableau 1 présente la répartition des dessins en fonction du nombre de doigt représenté (exact ou inexact) en pourcentage. À

Tableau 1

Répartition des dessins (en pourcentage) en fonction de la capacité à représenter 5 doigts

\begin{tabular}{lc} 
& Fréquence de dessin en pourcentage \\
\hline Nombre de doigt incorrect (D-) & $46,7 \%$ \\
Nombre de doigt correct (D+) & $53,3 \%$ \\
\hline
\end{tabular}

Tableau 2

Répartition des dessins (en pourcentage) en fonction du niveau AQ: 6 de planification local $(\mathrm{P}-)$ ou global $(\mathrm{P}+)$

\begin{tabular}{lc}
\hline & $\begin{array}{c}\text { Fréquence de dessin } \\
\text { en pourcentage }\end{array}$ \\
\hline Dessins planifiés localement $(\mathrm{P}-)$ & $40,3 \%$ \\
Dessins planifiés globalement $(\mathrm{P}+)$ & $59,7 \%$ \\
\hline
\end{tabular}

cet âge, la majorité des enfants représente les cinq doigts sur le dessin de la main.

Le Tableau 2 présente la répartition en pourcentage des dessins $\mathbf{T 2}$ obtenus par planification locale ou globale.

L'analyse des stratégies de planification montre qu'un nombre important de dessins est réalisé à partir d'un tracé continu. La planification globale du tracé, indicateur de l'existence d'un modèle interne, est possible dès la cinquième année pour une majorité de sujets.

La lecture du Graphique 1 permet de visualiser la répartition des F1 stratégies graphiques observées au dessin de la main réalisé par des enfants âgés en moyenne âgés de 4 ans 9 mois. Croiser la capacité à planifier le dessin de la main et à reporter le bon nombre de doigts permet d'identifier le pourcentage d'enfants recourant à une stratégie élaborée, c'est-à-dire recourant à un modèle interne pour guider le tracé tout en respectant le bon nombre de doigts. Sur l'ensemble des 62 productions recueillies, 33,8 \% des dessins correspondent aux critères d'une stratégie élaborée.

La moyenne des scores au dessin, calculés à partir de cinq critères repris de Gracia-Bafalluy et Noël (2008), est de 2,4 avec un écart-type de 1,65. L'analyse de variance appliquée aux moyennes des scores de dessin montre qu'il existe un effet significatif du type de stratégie sur la qualité figurative du dessin.

Le Graphique 2 montre que cet effet traduit une relation positive $\quad$ F2 entre les capacités de planification globale et la qualité du dessin de la main. Par ailleurs, le graphique montre aussi qu'au sein des productions graphiques planifiées localement $(\mathrm{P}-)$, la qualité figurative du dessin de la main est meilleure lorsque les cinq doigts ont été représentés. Au seuil de 0,05, les tests post-hoc réalisés montrent que les productions $\mathrm{D}-\mathrm{P}-$ ont un score qualitatif de dessin significativement plus faible comparativement aux trois autres types de stratégies. Par opposition, le score des productions

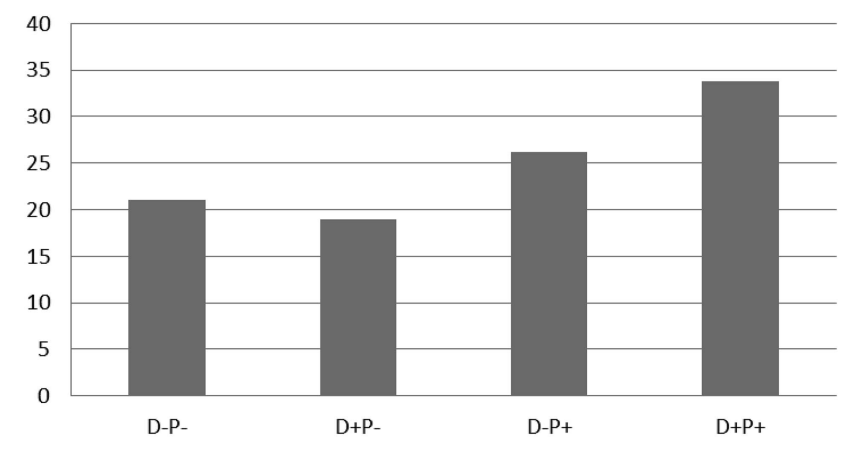

Figure 1. Fréquence d'occurrence (en pourcentage) des stratégies graphiques observées au dessin de la main. 
$F(1,60)=8,54 ; p<.001$

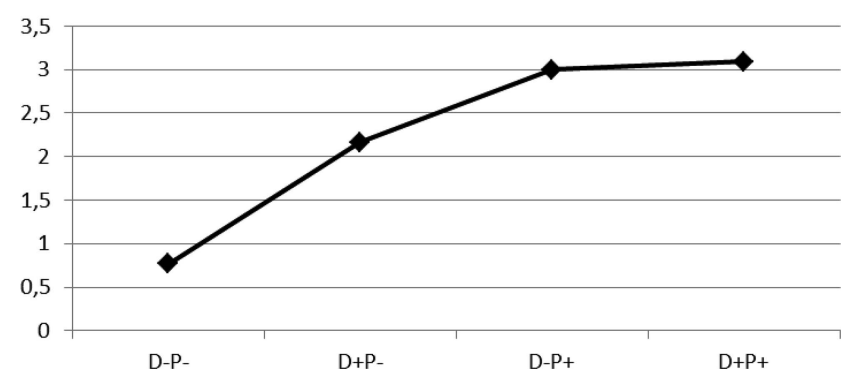

Figure 2. Relation entre stratégie graphique et score au dessin.

$\mathrm{D}+\mathrm{P}+$ tendent aussi à être meilleur que celui des productions $\mathrm{D}+\mathrm{P}-$.

Une analyse statistique implicative a été réalisée afin de vérifier un effet du sexe sur les variables nombre de doigts et planification. Les productions des garçons et des filles ne sont différenciables ni sur leur capacité de planification $(z=1,12 ;$ ns) ni sur leur capacité à représenter les 5 doigts $(z=0,14 ; n s)$. Enfin, l'analyse de variance réalisée sur le score global au dessin ne révèle pas d'effet du sexe, $F(1,60)=0,41$; ns.

\section{Caractérisation des habiletés numériques}

Comme les moyennes l'indiquent dans le Tableau 3, la capacité à mettre en correspondance terme à terme deux collections est réussie par $79 \%$ des enfants de l'échantillon. De la même façon, le principe de non-pertinence de l'ordre est observé chez 74,2\% des enfants de cet échantillon.

L'analyse statistique implicative ne permet de distinguer les productions des filles et des garçons ni sur la correspondance terme à terme $(\mathrm{z}=-0,19 ; p=0,95)$ ni sur le principe de non pertinence de l'ordre $(z=0,35 ; p=0,95)$.

\section{Relation entre dessin de la main et mesures des habiletés numériques}

Nous avons cherché à établir une éventuelle relation entre les stratégies au dessin de la main et les performances aux deux tâches numériques. Considérant que la stratégie $\mathrm{D}+\mathrm{P}+$ la plus élaborée correspond à une représentation interne de la main de qualité (une unité cognitive constituée d'une paume et de cinq doigts), nous étudierons la relation entre dessin de la main et habiletés numériques en comparant cette stratégie aux trois autres. Ainsi, lors de l'analyse statistique implicative, nous avons regroupé des stratégies les moins élaborées $(\mathrm{D}-\mathrm{P}-; \mathrm{D}+\mathrm{P}-; \mathrm{D}-\mathrm{P}+$ seront ici mentionnés comme $\mathrm{S}$-) pour les comparer à la stratégie la plus élaborée $(\mathrm{D}+\mathrm{P}+$ mentionnée comme $\mathrm{S}+)$.

Tableau 3

Pourcentage de réussite aux tâches numériques

\begin{tabular}{ccc}
\hline \multicolumn{3}{c}{ Pourcentage de réussite aux tâches numériques } \\
\hline \multirow{3}{*}{ C. Terme à terme } & Non-pertinence de l'ordre \\
\hline $79 \%$ & $74,2 \%$ \\
\hline
\end{tabular}

Tableau 4

Comparaison statistique des scores au dessin en fonction de la réussite ou non aux tâches numériques

\begin{tabular}{lcc}
\hline & $\begin{array}{c}\text { Correspondance } \\
\text { terme à terme }\end{array}$ & $\begin{array}{c}\text { Non-pertinence } \\
\text { de l'ordre }\end{array}$ \\
\hline $\begin{array}{l}\text { Score moyen (écart-type) pour } \\
\text { les élèves ayant réussi }\end{array}$ & $2(1,73)$ & $1,87(1,82)$ \\
$\begin{array}{l}\text { Score moyen (écart-type) pour } \\
\text { les élèves ayant échoué }\end{array}$ & $2,51(1,63)$ & $2,58(1,52)$ \\
$\begin{array}{l}\text { Valeurs } F \\
\text { Seuil de probabilité }\end{array}$ & $F(1,60)=2,24$ & $F(1,60)=0,97$ \\
& $p=0,14$ & $p=0,32$ \\
\hline
\end{tabular}

Le Tableau 4 permet d'analyser l'effet de la réussite aux deux T4 tâches numériques sur la qualité figurative du dessin. Les tests ne sont pas significatifs, il n'est donc pas permis d'établir un lien entre la qualité du dessin et la réussite aux deux tâches numériques considérées dans cette étude.

Le Graphique 3 met en évidence la relation entre le type de $\mathbf{F 3}$ stratégie utilisée et la capacité de l'enfant à réussir la tâche de correspondance terme à terme. On observe pour les stratégies les moins élaborées un nombre de réussites à la correspondance terme à terme supérieur au pourcentage d'échecs. Concernant les stratégies plus élaborées, on note que la réussite à la tâche de correspondance terme à terme est quasi systématique (un seul cas d'échec). À partir d'une analyse statistique implicative, nous pouvons établir, avec un seuil à la limite de la significativité, que la nature de la stratégie implique la réussite à la tâche numérique de correspondance terme à terme $(z=-1,62 ; p=0,052)$. Cette quasi-significativité s'explique par la rareté des cas pour lesquels une stratégie graphique élaborée serait associée à un échec à la tâche de correspondance terme à terme. En conformité avec l'hypothèse d'une relation implicative orientée, l'ASI réalisée pour la relation inverse (influence de la réussite en correspondance terme à terme sur la stratégie au dessin) ne permet pas de conclure à une relation significative ni même à une tendance $(z=-0,59$; $p=0,28)$.

Le Graphique 4 met en évidence la relation entre le type de F4 stratégie utilisée et la capacité de l'enfant à réussir la tâche de non-pertinence de l'ordre. On remarque pour les stratégies les moins élaborées un nombre de réussites à la tâche de nonpertinence de l'ordre supérieur au pourcentage d'échecs. Concer-

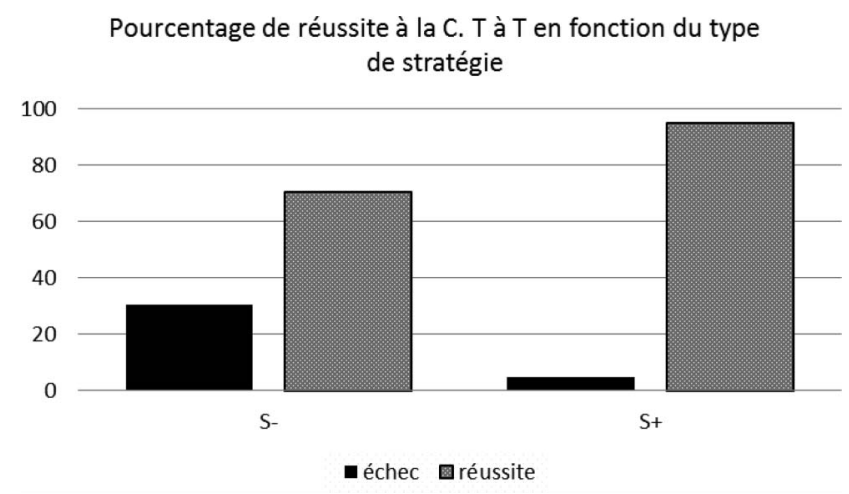

Figure 3. Relation entre type de stratégie et réussite à la correspondance terme à terme. 


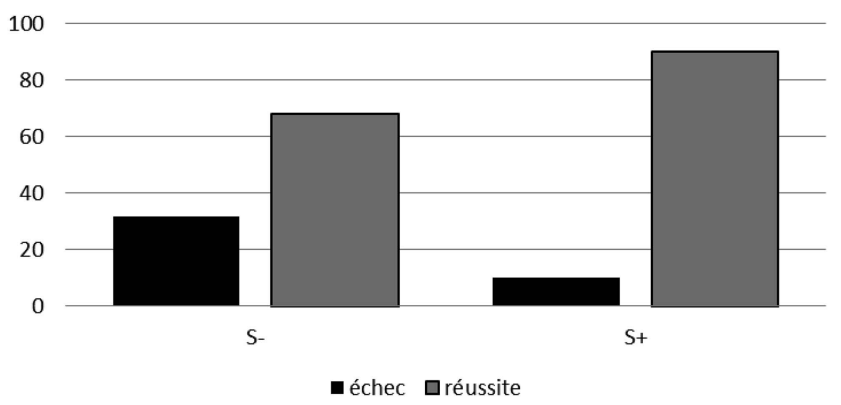

Figure 4. Relation entre type de stratégie et réussite à la tâche de non pertinence de l'ordre.

nant les stratégies plus élaborées, on note que la réussite à la tâche de non-pertinence de l'ordre est aussi fréquente (deux cas d'échec). À partir d'une analyse statistique implicative, nous ne pouvons pas établir que la nature de la stratégie tend à impliquer une réussite à la tâche numérique de non-pertinence de l'ordre $(z=-1,31 ; p=0,095)$. Une ASI réalisée pour tester la relation inverse (influence de la réussite aux épreuves numériques sur le dessin de la main) ne montre ni de relation significative ni de tendance $(z=-0,52 ; p=0,31)$ entre ces deux variables.

\section{Discussion}

Depuis une vingtaine d'années, les recherches en neuropsychologie, psychologie cognitive et psychologie développementale convergent pour attribuer un rôle prépondérant aux représentations digitales dans le développement des habiletés numériques. Néanmoins, ces relations restent obscures, probablement du fait que le choix des épreuves numériques et des indicateurs censés refléter les représentations digitales ne font pas consensus. En conclusion de leur chapitre, Marinthe, Fayol et Barrouillet (2001) concluaient sur l'existence d'une relation entre certaines représentations numériques, celles impliquant un format analogique, et les représentations digitales. Mais les auteurs de cette recherche déclaraient aussi qu'il restait à préciser comment s'établissent ces relations. En cas de difficulté en arithmétique, les auteurs se demandaient en quoi les représentations digitales pouvaient être déficitaires : « S'agit-il de représentations erronées ? De représentations floues ? » (p. 254). L'analyse de la littérature, et plus particulièrement d'une recherche de Gracia-Bafalluy et Noël (2008), nous a aussi conduit à nous interroger sur la pertinence de recourir au dessin comme indicateur de la représentation mentale de la main chez l'enfant. Notre étude se veut donc une contribution à l'étude des relations entre représentations mentales de la main et habileté numérique au travers du dessin de la main réalisé par l'enfant. Il s'agissait, dans une démarche exploratoire, de décrire les stratégies graphiques caractérisant le dessin de la main à un âge où les premières habiletés numériques se mettent en place. Nous avons pour cela basé notre analyse sur trois caractéristiques du dessin : la capacité de l'enfant à représenter cinq doigts, sa capacité à planifier l'ensemble de son dessin, et un score global reflétant la qualité figurative de la main. Par ailleurs, nous souhaitions savoir si certaines caractéristiques du dessin étaient associées à certaines habiletés numériques : l'une analogique, la correspondance terme à terme; l'autre plus conceptuelle, la non-pertinence de l'ordre.
Conformément à notre premier objectif, cette étude a permis de décrire les capacités des enfants de 4 ans 9 mois à représenter graphiquement une main le plus fidèlement possible sans l'aide d'un modèle externe. Nous avons constaté qu'une majorité d'enfants étaient capables de représenter les cinq doigts et que plus de la moitié de l'échantillon était en capacité de planifier leur tracé. Rappelons, qu'en situation de copie de formes géométriques, les enfants de 5 ans observés par Vinter et Marot (2003) et Vinter et al. (2008) montraient principalement une stratégie de planification locale. Il est permis de penser que la tâche de copie d'un ensemble de rectangles est plus complexe que celle du dessin de la main au regard du modèle interne qui doit être produit pour guider l'activité. Cette différence pourrait expliquer pourquoi les enfants de notre échantillon, âgés en moyenne de 4 ans et 9 mois, recourent majoritairement à une planification globale. En nous inspirant de la démarche de Vinter et Marot (2003), nous avons ensuite catégorisé les productions graphiques en identifiant des stratégies révélatrices de la nature des représentations mentales et de la taille des unités cognitives disponibles. Nous avons constaté que la capacité de planification n'était pas nécessairement associée à la capacité de représenter les cinq doigts. Parallèlement, nous avons identifié, sur la période étudiée, un tiers d'enfants présentant une stratégie élaborée. Conformément au modèle de Vinter et Marot (2003) et de Vinter et al. (2008), cette stratégie refléterait l'existence d'un modèle interne de la main sous la forme d'une seule unité cognitive constituée d'une paume et de cinq doigts. L'étude simultanée de ces stratégies et de la qualité figurative du dessin mesurée par un score inspiré de Gracia-Bafalluy et Noël (2008) a permis d'observer un lien entre ces deux mesures. Lorsque la planification est globale, la qualité figurative du dessin est meilleure. Cette analyse a aussi permis de montrer que les productions graphiques des enfants qui ne planifient pas et ne représentent pas cinq doigts sont significativement moins réussies, du point de vue figuratif, que celles des trois autres groupes. Ce résultat renforce l'idée défendue par Vinter et Marot (2003) selon laquelle l'étude du dessin, dans ses aspects figuratifs et syntaxiques, « constitue un terrain d'investigation tout à fait approprié pour approcher la question de l'évolution des connaissances chez l'enfant » (p. 105). Notre première hypothèse supposait que l'étude conjointe des aspects figuratifs et syntaxiques du dessin de la main permettrait de distinguer, au sein d'un échantillon d'enfants, une diversité de stratégies reflétant la nature des représentations mentales de la main. La diversité des stratégies observées et la cohérence observée entre les mesures figuratives et syntaxiques nous encouragent à valider cette première hypothèse. Finalement, rappelons aussi que dans cette étude, l'ensemble de ces conclusions valent quel que soit le sexe de l'enfant.

Notre second objectif se voulait une contribution à l'étude des relations entre habiletés numériques et représentations de la main au travers de l'étude du dessin de la main. Nous nous sommes demandé s'il était possible d'établir une relation entre la réussite à certaines épreuves numériques et certaines caractéristiques du des$\sin$. Les épreuves numériques de correspondance terme à terme et de non-pertinence de l'ordre ont été choisies parce qu'elles sont accessibles aux enfants de la tranche d'âge étudiée, mais aussi parce qu'elles permettent de repérer des élèves fragiles du point de vue de la construction des propriétés cardinales et ordinales du nombre (Fayol, 2012; Geary \& Hoard, 2002; Van Nieuwenhoven, Grégoire \& Noël, 2008). Dans cette étude, les deux épreuves ont 
été correctement réussies par plus de deux tiers des sujets, indépendamment du sexe.

Concernant l'analyse du score figuratif au dessin, cet indicateur ne semble pas être un bon prédicteur des performances aux tâches numériques. Dans l'étude de Gracia-Bafalluy et Noël (2008), nous avions trouvé paradoxal que le score au dessin ne soit pas corrélé aux compétences gnosiques digitales avant entraînement et nous nous étions interrogé sur la pertinence d'utiliser le dessin pour l'investigation des représentations mentales de la main. Dans cette étude, le score au dessin était principalement calculé à partir de critères figuratifs. Notre recherche contribue à la compréhension de ce résultat en soulignant que l'aspect figuratif du dessin ne nous semble pas la mesure la plus pertinente à utiliser pour accéder aux représentations mentales de la main chez l'enfant. En revanche, lorsque nous nous sommes intéressés à la relation entre les stratégies graphiques et la réussite à la tâche numérique de correspondance terme à terme, les conclusions ont été différentes. L'analyse statistique implicative, particulièrement bien adaptée à notre problématique et à la nature de nos données, nous a permis de montrer, avec un seuil à la limite de la significativité, que les enfants qui présentaient une représentation mentale de la main globale et complète étaient ceux qui réussissaient le mieux l'épreuve de correspondance terme à terme. Ainsi, au regard de notre seconde hypothèse, il nous semble que l'étude du dessin de l'enfant dans la cinquième année en relation avec les performances aux épreuves numériques de type analogique alimente la thèse d'une relation fonctionnelle entre représentation manuelle et habiletés numériques. L'étude de l'organisation syntaxique du tracé comme révélateur de la nature des représentations mentales de la main renforce ici l'idée défendue par Di Luca et Pesenti (2011) de l'existence de racines sensori-motrices sur lesquelles pourraient se construire le concept de nombre. Comment comprendre la relation entre représentation de la main et correspondance terme à terme ? Il nous semble que deux hypothèses explicatives peuvent être avancées. Soit ces enfants ont une moins bonne représentation sensori-motrice de la main et, de ce fait, tendent moins fréquemment à accompagner le comptage verbal d'une correspondance terme à terme entre les mots comptés et les doigts levés comme le décrivent Di Luca et Pesenti (2011). Une seconde explication serait de considérer que la tâche de correspondance terme à terme nécessite de recourir à une représentation de la quantité de la collection témoin, représentation éventuellement codée sous un format sensori-moteur. À ce titre, deux stratégies ont été identifiées par Van Nieuwenhoven (1999) pour réaliser cette tâche numérique. La moins élaborée des deux consiste à faire correspondre à chaque élément de la collection témoin, un autre élément. La seconde, plus élaborée et plus économique en temps, consiste à dénombrer la collection témoin puis, à partir de son cardinal, à constituer une collection équivalente. Dans notre étude, les enfants qui ont une représentation fiable de la main ont presque tous réussi cette tâche de correspondance terme à terme. Pour approfondir cette question, il serait intéressant, dans une étude complémentaire, d'analyser les stratégies des enfants durant la tâche de correspondance terme à terme afin de savoir s'ils opèrent la correspondance unité par unité ou s'ils se fondent sur le cardinal.

Contrairement à l'épreuve de correspondance terme à terme, il n'a pas été possible d'établir une relation d'implication entre certaines caractéristiques du dessin et la tâche de non-pertinence de l'ordre. Cette épreuve teste les connaissances conceptuelles des propriétés cardinales et ordinales du nombre. Pour Baroody (1987), cette tâche de nature verbale et non procédurale reflète la richesse et la diversité des expériences de dénombrement des enfants : c'est en dénombrant de différentes manières des collections d'objets que les enfants découvrent que l'ordre dans lequel il compte la collection n'a aucun impact sur le cardinal de cette collection. L'enfant qui découvre cette notion ne confondra pas les aspects cardinal et ordinal du nombre, car il saura que chaque mot-nombre peut être attribué à n'importe quel objet de la collection. L'absence de relation statistique entre les caractéristiques du dessin et l'épreuve conceptuelle reste difficilement interprétable, mais renforce l'idée déjà établie dans la littérature (GraciaBafalluy \& Noël, 2008; Marinthe et al., 1999, 2001) que les représentations manuelles ont une fonction probablement plus importante au cours de tâches nécessitant la manipulation de quantités sous un format analogique. Au contraire, la tâche de non-pertinence de l'ordre ne nécessite probablement pas le recours aux représentations analogiques des quantités en jeu.

Les doigts constitueraient une première représentation sensorimotrice des quantités manipulées ou perçues. Parallèlement et simultanément, l'utilisation de la main et des doigts comme outil pour représenter symboliquement les quantités selon des codes culturellement institués faciliterait la mise en relation entre des formats analogiques et symboliques du nombre. Ainsi, les enfants les plus habiles sur le plan des représentations sensori-motrices de la main et des doigts seraient cognitivement mieux outillés pour se représenter des petites quantités sur lesquelles opérer des transformations, pour comparer des quantités ou encore pour assister le comptage verbal par correspondance terme à terme entre les mots nombres énoncés et les doigts levés. Néanmoins, nous considérons, comme Di Luca et Pesenti (2011) que les représentations du nombre sont plurimodalitaires et que, par conséquent, les représentations sensori-motrices ne sont pas les seuls outils cognitifs disponibles au cours du développement. C'est d'ailleurs ce qui peut expliquer que certains sujets qui présentent une stratégie graphique peu élaborée puissent néanmoins être en réussite sur les tâches numériques analogique ou conceptuelle.

L'échantillon constitué pour cette étude rassemblait des enfants tous venants et les analyses statistiques réalisées n'ont pu montrer d'effet du sexe sur les variables étudiées. Il nous semble qu'une façon de confirmer ces résultats encore fragiles serait de compléter cette étude par l'analyse de ces relations auprès d'un échantillon constitué d'enfants présentant des difficultés dans les premières habiletés numériques. En effet, dans notre étude, seuls 6 enfants sur 62 ont échoué aux deux épreuves numériques. Nos conclusions devraient donc être confrontées auprès d'un large échantillon d'enfants ayant échoué simultanément aux deux épreuves numériques. Par ailleurs, l'étude de ces relations devrait être entreprise en deçà et au-delà de la période étudiée (cette investigation est en cours chez des enfants âgés de 6 ans) afin d'identifier une éventuelle logique développementale. Enfin, si ces investigations sont poursuivies, elles devront contrôler la variable liée à la latéralité afin de vérifier son impact éventuel sur la qualité de la production graphique notamment.

Finalement, nous souhaitons souligner que certaines de nos conclusions s'accordent avec les propositions de Jordan et al. (2009). À partir de suivis longitudinaux d'enfants issus de milieux socio-économiques défavorisés, ces auteurs constatent que ceux qui développeront des difficultés ultérieurement en mathématiques 
sont aussi ceux qui, dans les niveaux préélémentaires, avaient tendance à ne pas utiliser spontanément leurs doigts. De ce fait, les auteurs engagent les professionnels à enseigner explicitement l'utilisation des doigts en maternelle. Le dessin, fréquemment utilisé par les enseignants pour faciliter la conceptualisation de certains domaines, comme celui du schéma corporel, pourrait-il constituer un moyen privilégié d'élaborer une représentation mentale précise de la main ? Par notre contribution, nous avons pu montrer que toutes les informations permettant de décrire l'évolution du dessin au cours de la période étudiée ne se valent pas et que les indicateurs les plus pertinents lorsqu'ils sont considérés conjointement semblent être le nombre de doigts représenté et la planification du dessin. Le score reflétant la qualité figurative globale de la production n'est pas apparu comme un indicateur pertinent du niveau de l'enfant dans le domaine numérique. Aux questions que se posaient Marinthe et al. (2001) sur les caractéristiques des représentations digitales d'enfants présentant des difficultés dans le domaine numérique, notre étude apporte, nous semble-t-il, quelques éléments de réponse. Certains enfants pourraient éprouver des difficultés à se représenter mentalement la main comme une unité constituée d'une paume et de cinq doigts à une période où les habiletés numériques s'installent. L'absence de planification traduirait une difficulté à activer des connaissances conscientes et explicites concernant un outil, la main, qui joue un rôle indéniable dans la cognition numérique. D'autres recherches, utilisant le dessin comme moyen d'investiguer la représentation mentale de la main, devraient être entreprises. Si le dessin présente des variantes culturelles (Picard \& Baldy, 2011) et si le développement moteur et cognitif est sensible aux pratiques culturelles (Dasen, 1988), alors l'étude du dessin de la main chez des enfants ayant peu l'occasion de dessiner pourrait contribuer à porter un éclairage sur la nature des représentations digitales et de leurs relations avec les habiletés numériques. Cette étude est en cours d'analyse.

\begin{abstract}
This study aims at studying the relationship between manual and digital representations and numerical skills through the drawing of a hand. Sixty-two children (ages from 4 years old to 5 years and 6 months old) were asked to draw a hand and to carry out 2 numerical tasks: first to produce 2 equivalent collections and then to use the order-irrelevance principle. The statistical implicative analysis shows that more elaborated graphic strategies more strongly imply success producing 2 equivalent collections.
\end{abstract}

Keywords: sensory-motor representation, hand, drawing, numerical abilities, children

\section{Références}

Andres, M., Michaux, N., \& Pesenti, M. (2012). Common substrate for mental arithmetic and finger representation in the parietal cortex. NeuroImage, 62, 1520-1528. http://dx.doi.org/10.1016/j.neuroimage.2012 .05 .047

Baroody, A. J. (1987). Children's mathematical thinking: A developmental framework for preschool, primary, and special education teachers. New York, É.-U.: Teachers College Press.

Butterworth, B. (1999). The mathematical brain. London, R.-U. : MacMillan.
Crollen, V., Seron, X., \& Noël, M. P. (2011). Is finger counting necessary for the development of arithmetic abilities? Frontiers in Psychology, 2, 242. http://dx.doi.org/10.3389/fpsyg.2011.00242

Dasen, P. R. (1988). Développement psychologique et activités quotidiennes chez des enfants africains. Enfance, 41, 3-23. http://dx.doi.org/ 10.3406/enfan.1988.2152

Dehaene, S., Piazza, M., Pinel, P., \& Cohen, L. (2003). Three parietal circuits for number processing. Cognitive Neuropsychology, 20, 487506. http://dx.doi.org/10.1080/02643290244000239

Di Luca, S., \& Pesenti, M. (2011). Finger numeral representations: More than just another symbolic code. Frontiers in Psychology, 2, 272. http:// dx.doi.org/10.3389/fpsyg.2011.00272

Fayol, M. (2012). L'acquisition du nombre. Paris, France : PUF.

Geary, D. C., \& Hoard, M. K. (2002). Learning disabilities in basic mathematics: Deficits in memory and cognition. Dans J. M. Royer (éd.), Mathematical cognition (pp. 93-115). Greenwich, É.-U.: Information Age Publishing.

Gelman, R., \& Meck, E. (1983). Preschoolers' counting: Principles before skill. Cognition, 13, 343-359. http://dx.doi.org/10.1016/00100277(83)90014-8

Gertsmann, J. (1940). Syndrome of finger agnosia, disorientation for right and left, agraphia and acalculia. Archives of Neurology and Psychiatry, 44, 398-408. http://dx.doi.org/10.1001/archneurpsyc.1940.02280080158009

Goodenough, F. (1926). L'intelligence d'après le dessin. Le test du bonhomme. Paris, France : PUF. (traduction française, 1957).

Gracia-Bafalluy, M., \& Noël, M. P. (2008). Does finger training increase young children's numerical performance? Cortex, 44, 368-375. http:// dx.doi.org/10.1016/j.cortex.2007.08.020

Gras, R., \& Kuntz, P. (2008). An overview of the statistical implicative analysis (SIA). Dans R. Gras \& F. Guillet (éds), Statistical implicative analysis (pp. 11-40). Berlin, Allemagne: Springer. http://dx.doi.org/ 10.1007/978-3-540-78983-3_1

Jordan, N. C., Kaplan, D., Ramineni, C., \& Locuniak, M. N. (2008). Development of number combination skill in the early school years: When do fingers help? Developmental Science, 11, 662-668. http://dx .doi.org/10.1111/j.1467-7687.2008.00715.x

Jordan, N. C., \& Levine, S. C. (2009). Socioeconomic variation, number competence, and mathematics learning difficulties in young children. Developmental Disabilities Research Reviews, 15, 60-68. http://dx.doi .org/10.1002/ddrr.46

Kinsbourne, M., \& Warrington, E. K. (1963). The developmental Gerstmann syndrome. Archives of Neurology, 8, 490-501. http://dx.doi.org/ 10.1001/archneur.1963.00460050040004

Luquet, G. H. (1927). Le dessin enfantin. Genève, Suisse : Delachaux et Niestlé.

Marinthe, C., Fayol, M., \& Barrouillet, P. (1999). Performances perceptivo-tactiles et performances arithmétiques chez le jeune enfant. Rééducation orthophonique, 199, 69-79.

Marinthe, C., Fayol, M., \& Barrouillet, P. (2001). Gnosies digitales et développement des performances arithmétiques. Dans A. Van Hout, C. Meljac \& J. P. Fischer (éds), Troubles du calcul et dyscalculies chez l'enfant (pp. 239-254). Paris, France: Masson.

Noël, M. P. (2005). Finger Gnosia: A predictor of numerical abilities in children? Child Neuropsychology: A Journal on Normal and Abnormal Development in Childhood and Adolescence, 11(5), 413-430.

Picard, D., \& Baldy, R. (2011). Le dessin de l'enfant et son usage dans la pratique psychologique. Développements, 10, 45-60. http://dx.doi.org/ 10.3917/devel.010.0045

Rourke, B. P. (1993). Arithmetic disabilities, specific and otherwise: A neuropsychological perspective. Journal of Learning Disabilities, 26, 214-226. http://dx.doi.org/10.1177/002221949302600402

Sato, M., \& Lalain, M. (2008). On the relationship between handedness and hand-digit mapping in finger counting. Cortex, 44, 393-399. http:// dx.doi.org/10.1016/j.cortex.2007.08.005 
Siegler, R. S., \& Shrager, J. (1984). Strategies choices in addition and subtraction: How do children know what to do? Dans C. Sophian (éd.), Origins in cognitive skills (pp. 229-293). Hillsdale, É.-U.: Erlbaum.

Van Nieuwenhoven, C. (1999). Le comptage. Vers la construction du nombre. Bruxelles, Belgique : De Boeck.

AQ: 3 Van Nieuwenhoven, C., Grégoire, J., \& Noël, M. P. (2001). Test diagnostic des compétences de base en mathématiques. Montreuil, France : Édition ECPA.

Vinter, A. (1999). How meaning modifies drawing behavior in children. Child Development, 70, 33-49. http://dx.doi.org/10.1111/1467-8624.00004

Vinter, A., \& Marot, V. (2003). Les activités de dessin chez l'enfant : un accès à l'étude de ses représentations mentales. Approche neuropsychologique de l'apprentissage chez l'enfant, 72, 95-106.
Vinter, A., Picard, D., \& Fernandes, V. (2008). Graphic syntax and representational development. Dans C. Lange-Kuttner \& A. Vinter (éds), Drawing and the non-verbal mind (pp. 139-157). London, R.-U.: Cambridge University Press. http://dx.doi.org/10.1017/CBO9780511489730 .008

Williams, B. P., \& Williams, R. S. (1995). Finger numbers in the GrecoRoman world and the early middle ages. Isis, 86, 587-608. http://dx.doi .org/10.1086/357319

Reçu le 31 mars 2014

Révision reçue le 7 novembre 2014

Accepté le 26 janvier 2015 